Admiral Nurse palliative care approach against the domains and recommendations as set out in the EAPC white paper.

\section{P-171 CARING HEARTS: TRANSFORMING PALLIATIVE CARE FOR PATIENTS WITH HEART FAILURE}

${ }^{1}$ Louise Franklin, ${ }^{2}$ Jane Powell, ${ }^{1}$ Katherine Birch. ${ }^{1}$ Compton Care, Wolverhampton, UK; ${ }^{2} T$ The Royal Wolverhampton NHS Trust, Wolverhampton, UK

\subsection{6/bmjspcare-2018-hospiceabs. 196}

Over half a million people in the UK are currently living with heart failure (Hospice UK, 2017). It is a chronic condition characterised by frequent distressing symptoms and an unsure disease trajectory. People with heart failure, and those family and friends who care for them, have a high burden of unmet palliative care needs, but have poor access to hospice services (Hospice UK, 2017).

It is recognised that providing timely and effective care for those with heart failure is challenging. 'Looking after end-stage heart failure is not rocket science. It is much more difficult. The trajectory of a rocket is predictable: the course of heart failure is not. Patients are not made of metal, and it matters what happens to them' (Johnson, Lehman \& Hogg, 2015).

During 2017/2018 a large acute trust in the West Midlands, along with a palliative and end of life care provider, worked together to develop an innovative project to improve the lives of people living with heart failure. Funding was secured from St James's Place Charitable Foundation and the project commenced in January 2018.

The project aims to develop collaborative and creative approaches to delivering care and support for those with heart failure across the locality, and thus transform care for this group of patients and their families. This paper explores the approach taken (drawing on the work of Ind \& Watt, 2004, regarding harnessing staff potential), discusses the key phases within the project and presents some initial findings and insights from the project. These early outcomes focus primarily on referrals, patients' experiences of care and professional development. We also reflect on the learning which can be applied to any project involving collaboration within and across professional and organisational boundaries and consider the need for such approaches given the wider challenges facing health and social care.

\section{P-172 INTEGRATION OF PALLIATIVE CARE IN THE MANAGEMENT OF ADVANCED LIVER DISEASE} ${ }^{1,2}$ Nikki Reed, ${ }^{2}$ Alexandra Daley. 'Marie Curie Hospice West Midlands, UK; ${ }^{2}$ University
Hospitals of Birmingham NHS Foundation Trust (HGS), Birmingham, UK

\subsection{6/bmjspcare-2018-hospiceabs.197}

Background Between 1970 and 2010 the mortality rate for people dying with liver disease increased by $400 \%$. It is now the third most common cause of death in working-aged adults (18-65 years). This has resulted in a significant pressure on acute hospital services. Most patients with decompensated cirrhosis rarely receive palliative care intervention despite the significant symptom burden. It has been recognised that palliative care intervention improves symptoms, mood and quality of life in patients with advanced liver disease.
Aim To improve local access to palliative care services for patients with advanced non-malignant liver disease.

Method In early 2017 collaborative working between the hospice-based Consultant in Palliative Care and the acute hospital-based Consultant Gastroenterologist commenced. In October 2017 a combined liver clinic was commenced at the hospice with patients being reviewed by both gastroenterology and palliative care.

Results Although the monthly liver clinic is still in a very early phase our SystmOne data has demonstrated:

- Three patients with advanced non-malignant liver disease were referred for our palliative care services in the two years 2015-2016

- 15 patients with advanced non-malignant liver disease referred for our palliative care services for the two years 2017-2018 (still six months to go).

The majority of these referrals have been for outpatient clinic, however, direct referrals to the inpatient unit have increased as well.

Conclusion The increase in our referral numbers correlates with the start of our collaboration with the acute gastroenterology team. The need to improve end of life care for patients with liver disease has been recognised internationally. The huge symptom burden that these patients experience needs specialist palliative care input. We hope that our local collaboration will continue to help to address the inequality of access to our services for this group of patients.

\section{P-173 HOSPICE VOLUNTEERS SUPPORTING THOSE WITH NEUROLOGICAL CONDITIONS AND COGNITIVE CHANGE}

Marjolein Cleaver. LOROS, Leicester, UK

10.1136/bmjspcare-2018-hospiceabs. 198

The EVOLVE project was set up following a high number of referrals of patients with the dual diagnosis of MND and frontotemporal dementia. It was found that existing services did not adequately meet their needs. The EVOLVE project was made possible through funding from St James Place Charitable Foundation managed by Hospice UK to provide rehabilitative palliative care over a period of 18 months.

The project aimed to recruit and train volunteers, with a background in healthcare, to support patients with complex needs. Specially trained volunteers supported patients with a dual diagnosis of a neurological condition and cognitive change to work towards their own goals during regular home visits. At the same time these visits would provide respite for carers who were encouraged to use this time to attend to their own priorities.

Preliminary findings have shown that the calibre of volunteers recruited for the project has been very high with a high level of experience in caring. This has provided former carers with a volunteering role which they have enjoyed carrying out. Goal setting with patients proved difficult due to the cognitive changes patients experience, however, meaningful relationships were built up and social advantages were seen as significant. Carers seem to have benefitted most from this project through having an additional source of support, dedicated to their loved one's specific needs, who provided practical and emotional support. This project has enhanced clientcentred care as the volunteers provided an additional support 